\title{
Antifungal effect of voriconazole on intracellular Candida glabrata, Candida krusei and Candida parapsilosis in human monocyte-derived macrophages
}

\author{
Lawrence H. Bopp, ${ }^{1}$ Aldona L. Baltch, ${ }^{1,2}$ William J. Ritz, ${ }^{1,2}$ \\ Phyllis B. Michelsen ${ }^{1,2}$ and Raymond P. Smith ${ }^{1,2}$ \\ Infectious Disease Research Laboratory, Stratton Veterans Affairs Medical Center ${ }^{1}$, and Albany \\ Medical College ${ }^{2}$, Albany, NY 12208, USA
}

Correspondence

Aldona L. Baltch

aldona.baltch@med.va.gov

Received 1 November 2005

Accepted 22 March 2006

\section{INTRODUCTION}

Candidaemia caused by Candida albicans and other Candida species is known to be an important cause of morbidity and mortality, especially in debilitated and/or immunocompromised hosts (Anaissie et al., 1998; McNeil et al., 2001). Treatment of invasive tissue infections caused by Candida, including osteomyelitis, endocarditis, endophthalmitis and hepatic candidiasis, can be difficult and prolonged, and frequently results in recurrence or failure (Anaissie et al., 1998; McNeil et al., 2001). The development of resistance to fluconazole among C. albicans, Candida glabrata, Candida parapsilosis and other Candida species, as well as the innate resistance of Candida krusei to fluconazole, has made the treatment of these infections increasingly difficult (Loeffler \& Stevens, 2003; Ostrofsky-Zeichner et al., 2003; Pfaller et al., 2002). Furthermore, serious infections caused by species other than $C$. albicans, especially $C$. glabrata, C. parapsilosis and C. krusei, have increased in

Abbreviation: MDM, human monocyte-derived macrophage. hospitalized patients (Bodey et al., 2002; Ostrofsky-Zeichner et al., 2003).

Voriconazole, a novel triazole antifungal agent (Sanati et al., 1997; Sheehan et al., 1999), is known to be active against Candida species, including those resistant to fluconazole. Since phagocytic killing of Candida species by human neutrophils and monocytes/macrophages is the major host defence in serious fungal infections (Maródi et al., 1991a, b), it is important to demonstrate the activity of voriconazole against intracellular Candida in phagocytic cells. It is known that fluconazole can enter phagocytes (Pasqual at al., 1993), and the intracellular activity of voriconazole against $C$. albicans in phagocytes has already been demonstrated (Vora et al., 1998; Baltch et al., 2001, 2005). However, there is limited knowledge about its intracellular activity against $C$. glabrata, C. parapsilosis and C. krusei.

Our study describes the growth characteristics of intracellular fluconazole-resistant C. glabrata, C. parapsilosis and C. krusei in untreated human monocyte-derived macrophages (MDMs), and the effect of increasing concentrations 
of voriconazole on the intracellular growth and survival of these Candida species. It also provides data suggesting that maximal effective intracellular concentrations of voriconazole can be reached in these phagocytic cells.

\section{METHODS}

Yeast strains. All yeast strains used in this study were clinical isolates. Four strains each of C. glabrata, C. krusei and C. parapsilosis were tested. C. krusei strain 6 and C. parapsilosis strain 25 were obtained from the University of Iowa, Iowa City, IA. C. krusei strains 1, 2 and 4, C. glabrata strains 11, 12, 14 and 20, and C. parapsilosis strains 21, 29 and 30 were obtained from the Wadsworth Center, New York State Department of Health, Albany, NY. These strains were chosen because they are moderately resistant to fluconazole (MICs ranging from 16 to $64 \mu \mathrm{g} \mathrm{ml}^{-1}$ ) and are susceptible to voriconazole. The Clinical Microbiology Laboratory at the Stratton Veterans Affairs Medical Center verified the species of each yeast strain using the RapID Yeast Plus Identification System (Remel) and microscopic examination of yeast grown on corn meal agar. For preparation of inocula, Candida from stock cultures frozen at $-80^{\circ} \mathrm{C}$ in $15 \%(\mathrm{v} / \mathrm{v})$ glycerol were streaked for isolation on Sabouraud dextrose (SD) agar and incubated overnight at $37^{\circ} \mathrm{C}$. Four to five well-isolated colonies from a plate were used to make a suspension in sterile $0.85 \%(\mathrm{w} / \mathrm{v}) \mathrm{NaCl}$. The absorbance at $530 \mathrm{~nm}$ was determined and adjusted to yield a final cell density of approximately $2 \times 10^{6}$ c.f.u. $\mathrm{ml}^{-1}$. The cells were then diluted 100 -fold to $2 \times 10^{4}$ c.f.u. $\mathrm{ml}^{-1}$. The cell density of each inoculum was verified using the standard plate count method.

Antimicrobial compounds and MIC testing. Laboratorystandard powders of voriconazole and fluconazole were provided by Pfizer. Voriconazole and fluconazole solutions were prepared according to the supplier's directions and filter-sterilized $(0.45 \mu \mathrm{m}$ pore-size) immediately prior to use. MICs of voriconazole and fluconazole for all Candida strains used in this study were determined in triplicate using the National Committee for Clinical Laboratory Standards-approved macrodilution method M27-A2 (National Committee for Clinical Laboratory Standards, 2002). MICs (in $\mu \mathrm{g} \mathrm{ml}^{-1}$ ) of fluconazole/voriconazole for C. glabrata strains 11 , 12,14 and 20 were $32 / 1,16 / 0 \cdot 5,64 / 2$ and 16/1, respectively. For $C$. krusei strains 1, 2, 4 and 6 , MICs (in $\mu \mathrm{g} \mathrm{ml}^{-1}$ ) of fluconazole/voriconazole were $16 / 0 \cdot 5,16 / 0 \cdot 5,64 / 0 \cdot 5$ and $32 / 0 \cdot 25$, respectively. For $C$. parapsilosis strains $21,25,29$ and 30 , MICs (in $\mu \mathrm{g} \mathrm{ml}^{-1}$ ) of fluconazole/voriconazole were $8 / 0 \cdot 25,32 / 0 \cdot 5,64 / 1$ and $16 / 0 \cdot 5$, respectively.

Human monocytes. Monocytes were obtained from heparinized blood of healthy human donors who had signed the informedconsent form approved by the Institutional Review Board of the Stratton Veterans Affairs Medical Center. Mononuclear cells were separated from whole blood by using Histopaque 1077 (Sigma). The resulting mononuclear cell preparation was more than $98 \%$ pure. The separated cells were resuspended at a concentration of $2 \times 10^{6}$ cells $\mathrm{ml}^{-1}$ in RPMI + [RPMI 1640 medium (Sigma) supplemented with $15 \%$ heat-inactivated fetal bovine serum (F-2442, Sigma)]. Cell viability, determined by the trypan blue exclusion test, was at least $98 \%$.

Time-kill studies. Time-kill assays were run for voriconazole. Mononuclear cells prepared as described above were added to the wells of 48-well culture plates (Corning/Costar) at $10^{6}$ cells per well and allowed to adhere for $2 \mathrm{~h}$. Monocytes adhered to the wells in contiguous monolayers, while non-adherent cells, including lymphocytes, were removed by aspiration. The adhered cells were considered to be MDMs. A suspension of Candida cells in RPMI + $(500 \mu \mathrm{l}$ at $2 \times 10^{4}$ c.f.u. $\mathrm{ml}^{-1}$ ) was then added to each well. Cell monolayers were incubated for $1 \mathrm{~h}$ at $35^{\circ} \mathrm{C}$ in an atmosphere containing $5 \%$ $\mathrm{CO}_{2}$ in order to allow phagocytosis of the yeast to occur. Following phagocytosis, the medium was removed by aspiration and the MDM monolayers were washed once with RPMI + (additional washing did not further decrease the number of yeast in the well). The intracellular location of the yeast was verified by differential fluorescent staining of intracellular and extracellular yeast and examination by fluorescence microscopy. Following phagocytosis and washing, approximately $5 \times 10^{3}$ intracellular yeast per well remained. After washing, RPMI + containing voriconazole at concentrations from $0 \cdot 1$ to $5 \times$ MIC was added. Incubation was at $35^{\circ} \mathrm{C}$ in an atmosphere containing $5 \% \mathrm{CO}_{2}$. Following incubation $(0,24$ and $48 \mathrm{~h}$ ), the medium was removed from each well by aspiration and the MDMs were lysed using sterile distilled water in order to release the yeast. The number of viable yeast in each sample was determined by using SD agar and the standard plate count method. In each experiment, each experimental condition was run in duplicate (two wells) and the number of surviving yeast in each well was determined in duplicate. Each assay was repeated at least three times. Kill curves represent geometric means of the numbers of surviving organisms at 24 and $48 \mathrm{~h}$, expressed as a percentage of the c.f.u. $\mathrm{ml}^{-1}$ at $0 \mathrm{~h}$ (number of organisms at 24 or $48 \mathrm{~h}$ divided by the number of organisms for the same experimental condition at $0 \mathrm{~h}$, multiplied by $100)$. It should be noted that since the ordinates of these graphs are logarithmic, differences that appear small may still be significant. For time-kill curves, inhibition is defined as the number of viable yeast being less than the number in the untreated control but greater than the number at $0 \mathrm{~h}$, and killing (fungicidal activity) is defined as the number of viable yeast being lower than the number at $0 \mathrm{~h}$.

Statistical methods. The analysis of variance (Stuart \& Ord, 1991a) was used to analyse the observed c.f.u. $\mathrm{ml}^{-1}$ transformed by $\log _{10}$. Post hoc comparisons were made under the Dunn procedure (Stuart \& Ord, 1991b). For presentation of results, the mean $\log _{10}$ values were converted to the geometric mean numbers of c.f.u. $\mathrm{ml}^{-1}$, expressed as a percentage of the geometric mean c.f.u. $\mathrm{ml}^{-1}$ at $0 \mathrm{~h}$. Regression curves were fitted to the observed data using our adaptation of the von Bertalanffy model (Draper \& Smith, 1981a) and the least squares method (Draper \& Smith, 1981b). The regression equation used was:

$y=m+10^{a+b x+c x^{2}}$

where $y$ is the percentage of viable counts at 24 or $48 \mathrm{~h}$ (i.e. geometric mean number of c.f.u. $\mathrm{ml}^{-1}$ at 24 or $48 \mathrm{~h}$, expressed as a percentage of the geometric mean number of c.f.u. $\mathrm{ml}^{-1}$ at $\left.0 \mathrm{~h}\right), \mathrm{m}$ is the minimum percentage viable count attainable, and $x$ is the concentration of voriconazole, expressed as a multiple of the MIC. The parameters $a, b$ and $c$ determine the configuration of the exponential curve, which depicts the percentage viable counts as a function of the voriconazole concentration, specific for each Candida species. The level of significance was $0 \cdot 05$.

\section{RESULTS}

\section{Growth and survival of Candida in untreated MDM}

Fig. 1 and Table 1 demonstrate that the growth and survival of intracellular yeast in untreated controls were specific for each Candida species tested. The greatest increase in the viable count at $48 \mathrm{~h}$ was observed for C. parapsilosis (28 $121 \%$ ), while C. glabrata increased to $351 \%$ during the same period. In contrast, there was a continuous decline in 

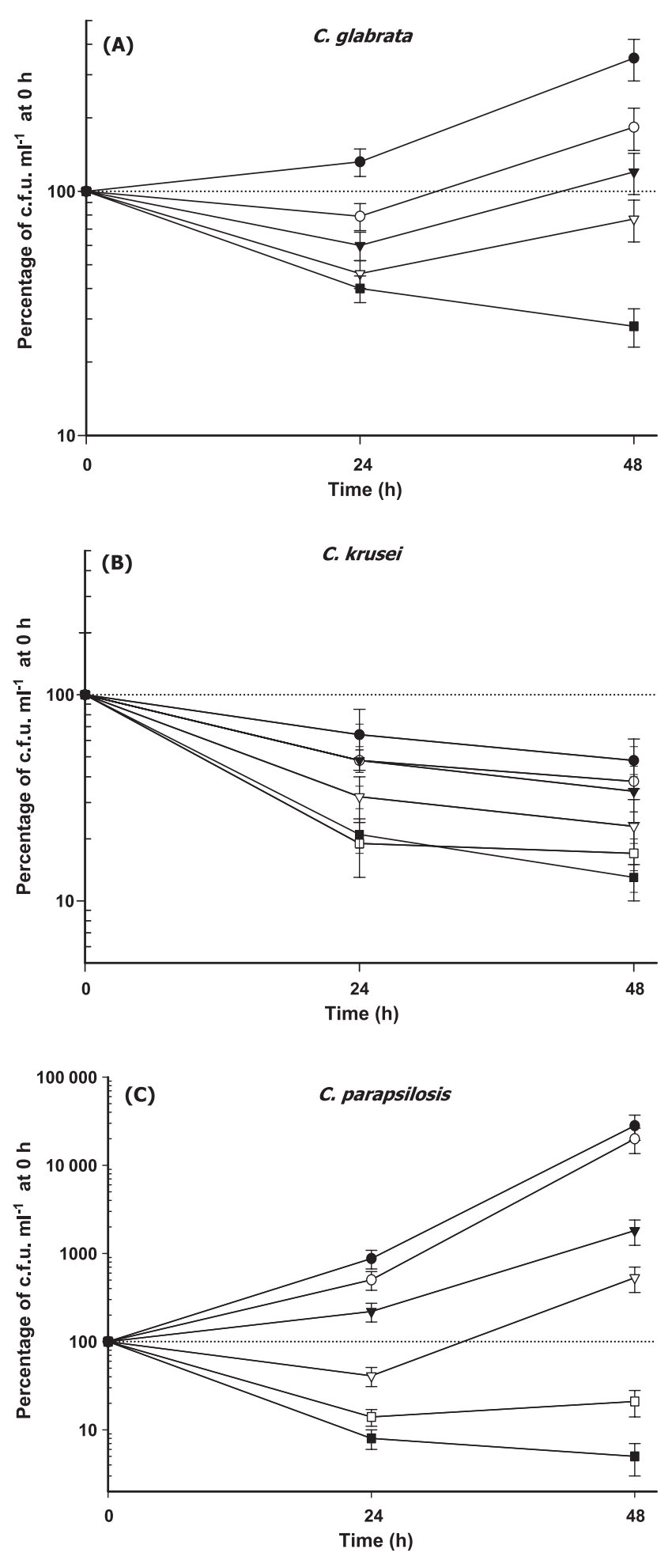

Fig. 1. Intracellular killing of C. glabrata (A), C. krusei (B) and C. parapsilosis (C) by voriconazole in MDMs. The geometric means are shown of four strains per species and three assays per strain expressed as a percentage of the c.f.u. $\mathrm{ml}^{-1}$ at $0 \mathrm{~h}$. -, control; $\bigcirc, 0.1 \times \mathrm{MIC}$ voriconazole; $\mathbf{\nabla}, 0.5 \times \mathrm{MIC}$ voriconazole; $\nabla, 1 \times$ MIC voriconazole; $\square, 2.5 \times$ MIC voriconazole; 口, $5 \times$ MIC voriconazole. For all figures, the error bars represent $1 \times$ SEM, and the dotted line at $100 \%$ represents the number of viable organisms at $0 \mathrm{~h}$.
Table 1. Effect of voriconazole on growth and survival of three Candida species in MDMs

Viable count values are geometric means of c.f.u. $\mathrm{ml}^{-1}$ at $48 \mathrm{~h}$ expressed as percentages of c.f.u. $\mathrm{ml}^{-1}$ at $0 \mathrm{~h}$.

\begin{tabular}{|lc|}
\hline Candida species and treatment & Viable counts \\
\hline C. glabrata & \\
Untreated & 351 \\
$5 \times$ MIC voriconazole & 28 \\
C. krusei & \\
Untreated & 42 \\
$5 \times$ MIC voriconazole & 13 \\
C. parapsilosis & \\
Untreated & 28121 \\
$5 \times$ MIC voriconazole & 5 \\
\hline
\end{tabular}

the number of viable C. krusei over $48 \mathrm{~h}$ to $42 \%$ of the number initially present.

\section{Effect of voriconazole on growth and survival of Candida in MDM}

Fig. 1(A) shows the combined results of time-kill assays for four strains of C. glabrata. Compared to the untreated control, voriconazole had significant intracellular activity against $C$. glabrata at all concentrations tested $(P<0 \cdot 05)$, and this activity was concentration dependent. At $24 \mathrm{~h}$, the number of surviving yeast was significantly lower than the number at $0 \mathrm{~h}(100 \%)$ at all concentrations except $0 \cdot 1 \times$ MIC $(P<0 \cdot 05)$. At $48 \mathrm{~h}$, there was inhibition at $0 \cdot 1 \times$ MIC $(P<0 \cdot 05)$. At 0.5 and $1 \times$ MIC at $48 \mathrm{~h}$, the numbers of surviving yeast were not significantly different from the numbers at $0 \mathrm{~h}$. However, the values were significantly lower than in the untreated controls $(P<0 \cdot 05)$. At $5 \times$ MIC and $48 \mathrm{~h}$, there was fungicidal activity against $C$. glabrata $(P<0 \cdot 05)$.

Results for C. krusei are shown in Fig. 1(B). At 24 h, voriconazole had significant intracellular activity against $C$. $k r u s e i$ at all concentrations tested $(P<0 \cdot 05)$, and the activity was concentration dependent. The numbers of viable organisms at $24 \mathrm{~h}$ for $0 \cdot 1$ and $0 \cdot 5 \times$ MIC were lower than for the control, but did not differ from one another. Numbers of viable yeast at $24 \mathrm{~h}$ for $1,2.5$ and $5 \times$ MIC were lower than they were for $0 \cdot 1$ or $0.5 \times$ MIC, and numbers of surviving organisms for 2.5 and $5 \times$ MIC were significantly lower than for $1 \times$ MIC $(P<0 \cdot 05)$. At $48 \mathrm{~h}$, all of the viable counts, including those for the untreated control, were significantly lower than they were at $0 \mathrm{~h}(P<0 \cdot 05)$, and decreased as the voriconazole concentration increased. Fungicidal activity was not different for the untreated control, $0 \cdot 1$ or $0.5 \times$ MIC of voriconazole at $48 \mathrm{~h}$. Activity was greater at $1,2 \cdot 5$ and $5 \times$ MIC than at $0,0 \cdot 1$ and $0 \cdot 5 \times \operatorname{MIC}(P<0 \cdot 05)$, but activities at $1,2 \cdot 5$ and $5 \times$ MIC did not differ significantly from one another. 
Fig. 1(C) shows the results for C. parapsilosis. At $24 \mathrm{~h}$, voriconazole had significant intracellular activity against C. parapsilosis at all concentrations except $0 \cdot 1 \times \mathrm{MIC}$, and the number of surviving yeast decreased with respect to the untreated control as the voriconazole concentration increased $(P<0 \cdot 05)$. The number of surviving yeast at $24 \mathrm{~h}$ was significantly lower for 2.5 and $5 \times$ MIC than for $1 \times$ MIC $(P<0 \cdot 05)$, but the difference between $2 \cdot 5$ and $5 \times$ MIC was not significant. At $48 \mathrm{~h}$, the number of viable yeast was significantly less than for the untreated control at all voriconazole concentrations except $0 \cdot 1 \times$ MIC. There was inhibition at 0.5 and $1 \times$ MIC, but there was fungicidal activity only at $2 \cdot 5$ and $5 \times$ MIC.

Fig. 2 shows regression curves fitted to the observed (experimental) data shown in Fig. 1, as well as the observed data.
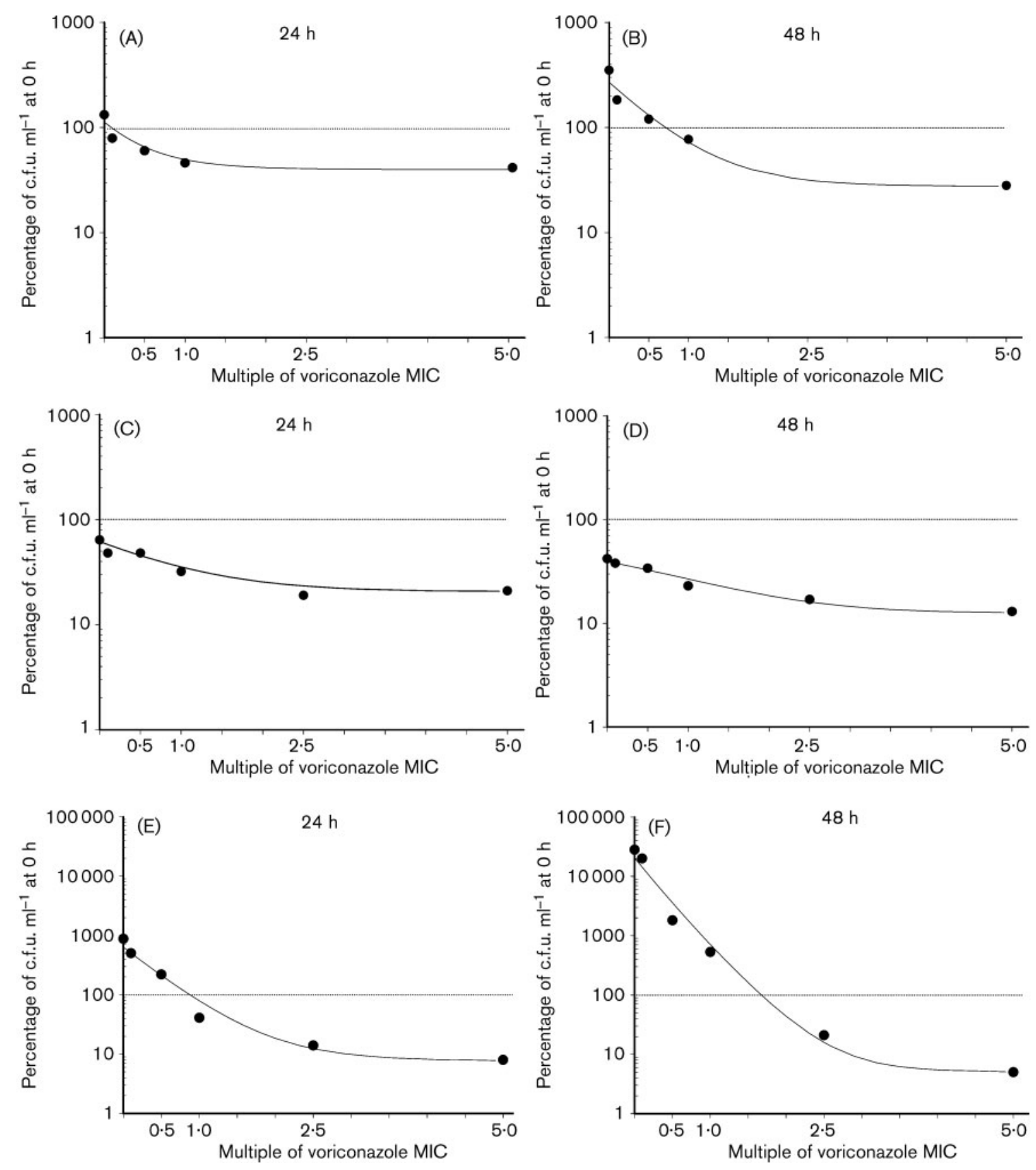

Fig. 2. Fitted regression curves and observed (experimental) data showing intracellular killing of Candida by voriconazole in MDMs. The geometric means are shown of four strains per species and three assays per strain, expressed as a percentage of the c.f.u. $\mathrm{ml}^{-1}$ at $\mathrm{O}$ h, at 24 and $48 \mathrm{~h}$ for C. glabrata (A, B), C. krusei (C, D) and C. parapsilosis (E, F) in MDMs. Solid lines, fitted regression curves; $\bullet$, observed (experimental) data. 
The fitted values, specific for assay hours 24 and 48, were calculated using the equation defined in Methods. The $r^{2}$ values ranged from 0.983 to 0.996 , indicating very close fit of the regression curves to the observed (experimental) data (Draper \& Smith, 1981b). Comparison of the fitted regression curves for the three Candida species at $48 \mathrm{~h}$ shows that in the absence of voriconazole, intracellular killing continued for C. krusei but not for C. glabrata or C. parapsilosis (Table 1, Fig. 2). Addition of voriconazole resulted in a dramatic decrease in the number of viable intracellular $C$. parapsilosis and, to a lesser extent, of C. glabrata and C. krusei. For C. parapsilosis, maximum intracellular fungicidal activity was reached at $4-5 \times$ MIC of voriconazole, and for C. glabrata and C. krusei, at 3.5-5 $\times$ MIC of voriconazole. For C. krusei, the addition of voriconazole enhanced intracellular killing, although the lowest percentage viable count attained was not as low as for C. parapsilosis (Table 1, Fig. 2).

\section{DISCUSSION}

Intracellular survival and ability to grow intracellularly in the absence and presence of voriconazole varied markedly among the three yeast species evaluated. C. krusei did not grow intracellularly, even in untreated controls. In contrast, during the same period, the number of viable C. glabrata increased slightly, while the number of viable $C$. parapsilosis increased considerably more (Table 1, Fig. 2). Our data from previous studies with C. albicans shows that C. albicans grows more rapidly in MDMs than these three species, especially during the first $24 \mathrm{~h}$, but that at $48 \mathrm{~h}$, the number of c.f.u. $\mathrm{ml}^{-1}$ is similar to that of C. parapsilosis (Baltch et al., 2001, 2005). Voriconazole $(5 \times$ MIC) had significant fungicidal activity against all three species, with the greatest intracellular fungicidal activity against $C$. parapsilosis, the most rapidly growing of the three species tested. When treated with voriconazole at $5 \times \mathrm{MIC}$, the number of viable C. parapsilosis at $48 \mathrm{~h}$ was only $5 \%$ of that at $0 \mathrm{~h}$, compared to $13 \%$ for C. krusei and $28 \%$ for C. glabrata. Thus, among these Candida species, a relatively high (or low) viable count in the absence of voriconazole did not imply a relatively high (or low) viable count in the presence of the most effective concentration of voriconazole.

In this study, the availability of abundant data for each species (four strains, three separate experiments, each experimental condition done in duplicate in each experiment) allowed the development of regression curves showing the relationship between voriconazole concentration (expressed as a multiple of the MIC) and intracellular killing over time. To our knowledge, this is the first application of this type of mathematical description of time-kill data for Candida species. Such analyses can be useful, because they demonstrate the level of intracellular killing at any voriconazole concentration, specific for time, and suggest the maximum level of killing possible for each Candida species. Since maximum intracellular fungicidal activity against all 12 strains of the three Candida species studied was reached at approximately $3 \cdot 5-5 \times$ MIC, this could represent a threshold of activity, and increasing the extracellular voriconazole level beyond the threshold may have limited usefulness.

Although up to $95 \%$ killing of intracellular yeast was achieved with voriconazole, the regression curves clearly demonstrate that in our in vitro model, complete eradication of intracellular yeast is not likely to be achieved using voriconazole alone. Use of a triazole along with an appropriate cytokine (Baltch et al., 2001) and/or another antifungal drug with a different mode of action may be useful to enhance the killing of intracellular yeast. The potential utility of combination therapy against Candida infections has recently been reviewed (Mukhergee et al., 2005), and is supported by reports of efficacy in vitro against $C$. albicans (Ghannoum et al., 1995) and in vivo against fluconazoleresistant C. krusei and C. glabrata (Girmenia et al., 2003).

In conclusion, our data show that intracellular growth and survival of different Candida species in the absence or presence of voriconazole vary markedly. The activity of voriconazole depends on the concentration of the drug and the time of exposure. For the 12 strains of the three Candida species studied, the regression curves demonstrate the level of intracellular killing by MDMs, with or without voriconazole, at any drug concentration, and show that the maximum intracellular anticandidal activity of voriconazole was reached at $3 \cdot 5-5 \times$ MIC.

\section{ACKNOWLEDGEMENTS}

This work was supported by Pfizer Laboratories and in part by the Veterans Affairs Research Service. The authors thank Dr Vishnu Chaturvedi, Wadsworth Center, New York State Department of Health, Albany, NY, and Dr Michael Pfaller, University of Iowa, Iowa City, IA, for providing the Candida strains used in this study.

\section{REFERENCES}

Anaissie, E. J., Rex, J. H., Uzun, O. \& Vartivarian, S. (1998). Predictors of adverse outcome in cancer patients with candidemia. Am J Med 104, 238-245.

Baltch, A. L., Smith, R. P., Franke, M. A., Ritz, W. J., Michelsen, P. B. \& Bopp, L. H. (2001). Effects of cytokines and fluconazole on the activity of human monocytes against Candida albicans. Antimicrob Agents Chemother 45, 96-104.

Baltch, A. L., Smith, R. P., Ritz, W. J., Bopp, L. H. \& Michelsen, P. B. (2005). Intracellular activity of voriconazole, fluconazole, and itraconazole against Candida albicans in human monocytes with and without activation by GM-CSF and TNF- $\alpha$. J Appl Res 5, 543-552.

Bodey, G. P., Mardani, M., Hanna, H. A., Boktour, M., Abbas, J., Girgawy, E., Hachem, R. Y., Kontoyiannis, D. P. \& Raad, I. I. (2002). The epidemiology of Candida glabrata and Candida albicans fungemia in immunocompromised patients with cancer. Am J Med 112, 380-385.

Draper, N. \& Smith, H. (1981a). Applied Regression Analysis, 2nd edn, p. 512. New York: Wiley. 
Draper, N. \& Smith, H. (1981b). Applied Regression Analysis, 2nd edn, pp. 85-96. New York: Wiley.

Ghannoum, M. A., Fu, Y., Ibrahim, A. S., Mortara, L. A., Shafiq, M. C., Edward, J. E., Jr \& Criddle, R. S. (1995). In vitro determination of optimal antifungal combinations against Cryptococcus neoformans and Candida albicans. Antimicrob Agents Chemother 39, 2459-2465.

Girmenia, C., Venditti, M. \& Martino, P. (2003). Fluconazole in combination with flucytosine in the treatment of fluconazole-resistant Candida infections. Diagn Microbiol Infect Dis 46, 227-231.

Loeffler, J. \& Stevens, D. A. (2003). Antifungal drug resistance. Clin Infect Dis 36 (supplement 1), S31-S41.

Maródi, L., Korchak, H. M. \& Johnston, R. B., Jr (1991a). Mechanisms of host defense against Candida species. 1. Phagocytosis by monocytes and monocyte-derived macrophages. J Immunol 146, 2783-2789.

Maródi, L., Forehand, J. R. \& Johnston, R. B., Jr (1991b). Mechanisms of host defense against Candida species. 2. Biochemical basis for the killing of Candida by mononuclear phagocytes. J Immunol 146, 2790-2794.

McNeil, M. M., Nash, S. L., Hajjeh, R. A., Phelan, M. A., Conn, L. A., Plikaytis, B. D. \& Warnock, D. W. (2001). Trends in mortality due to invasive mycotic disease in the United States, 1980-1997. Clin Infect Dis 33, 641-647.

Mukhergee, P. K., Sheehan, D. J., Hitchcock, C. A. \& Ghannoum, M. A. (2005). Combination treatment of invasive fungal infections. Clin Microbiol Rev 18, 163-194.

National Committee for Clinical Laboratory Standards (2002). Reference Method for Broth Dilution Antifungal Susceptibility Testing of Yeasts. Approved standard. NCCLS document M27-A2. Wayne, PA: National Committee for Clinical Laboratory Standards.

Ostrofsky-Zeichner, L., Rex, R. H., Pappas, P. G. \& 9 other authors (2003). Antifungal susceptibility survey of 2,000 bloodstream Candida isolates in the United States. Antimicrob Agents Chemother 47, 3149-3154.

Pasqual, A., Garcia, I., Conejo, C. \& Perea, E. P. (1993). Uptake and intracellular activity of fluconazole in human polymorphonuclear leukocytes. Antimicrob Agents Chemother 37, 187-190.

Pfaller, M. A., Diekema, D. J., Jones, R. N., Messer, S. A., Hollis, R. J. \& the Sentry Participants Group (2002). Trends in antifungal susceptibility of Candida spp. isolated from pediatric and adult patients with bloodstream infections: SENTRY Antimicrobial Surveillance Program, 1997-2000. J Clin Microbiol 40, 852-856.

Sanati, H., Belanger, P., Fratti, R. \& Ghannoum, M. (1997). A new triazole, voriconazole (UK 109,496), blocks sterol biosynthesis in Candida albicans and Candida krusei. Antimicrob Agents Chemother 41, 2492-2496.

Sheehan, D. J., Hitchcock, C. A. \& Sibley, C. M. (1999). Current and emerging azole antifungal agents. Clin Microbiol Rev 12, 40-79.

Stuart, A. \& Ord, J. K. (1991a). Kendall's Advanced Theory of Statistics, vol. 2, pp. 1101-1153. New York: Oxford University Press.

Stuart, A. \& Ord, J. K. (1991b). Kendall's Advanced Theory of Statistics, vol. 2, p. 1149, section 29.58. New York: Oxford University Press.

Vora, S., Purimetla, N. \& Brummer, E. (1998). Activity of voriconazole, a new triazole, combined with neutrophils and monocytes against Candida albicans: effect of G-CSF and GM-CSF. Antimicrob Agents Chemother 42, 907-910. 